Research Paper

\title{
Circulating Matrix Metalloproteinase-2 and -9 Enzyme Activities in the Children with Ventricular Septal Defect
}

\author{
Kun-Shan Cheng1,2,t, Yan-Chiou Liao, ${ }^{1, \dagger}$, Mu-Yuan Chen ${ }^{1}$, Tang-Ching Kuan¹, Yi-Han Hong1, Li Ko1, \\ Wen-Yeh Hsieh1,3, Chien-Liang Wu ${ }^{3}$, Ming-Ren Chen ${ }^{4}$, Chih-Sheng Lin ${ }^{1,}$, \\ 1. Department of Biological Science and Technology, National Chiao Tung University, Hsinchu, Taiwan \\ 2. Division of Cardiology, Department of Pediatrics, Mackay Memorial Hospital, Hsinchu, Taiwan \\ 3. Division of Chest Medicine, Department of Internal Medicine, Mackay Memorial Hospital, Hsinchu, Taiwan \\ 4. Division of Cardiology, Department of Pediatrics, Mackay Memorial Hospital, Taipei, Taiwan \\ † Equal contribution as the first author
}

$\triangle$ Corresponding author: Chih-Sheng Lin, Department of Biological Science and Technology, National Chiao Tung University, No.75 Po-Ai Street, Hsinchu 30068, Taiwan. Tel.: +886-3-5131338; Fax: +886-3-5729288. E-mail: lincs@mail.nctu.edu.tw

(C) Ivyspring International Publisher. This is an open-access article distributed under the terms of the Creative Commons License (http://creativecommons.org/ licenses/by-nc-nd/3.0/). Reproduction is permitted for personal, noncommercial use, provided that the article is in whole, unmodified, and properly cited.

Received: 2013.04.04; Accepted: 2013.05.21; Published: 2013.06.12

\begin{abstract}
Ventricular septal defect (VSD) is the most common form of congenital heart diseases. Matrix metalloproteinases (MMPs) are a family of zinc-dependent endopeptidases involved in causal cardiac tissue remodeling. We studied the changes of circulating MMP-2 and MMP-9 activities in the patients with VSD severity and closure. There were 96 children with perimembranous VSD enrolled in this study. We assigned the patients into three groups according to the ratio of VSD diameter/diameter of aortic root (Ao). They were classified as below: Trivial (VSD/Ao ratio $\leq 0.2$ ), Small $(0.2<$ VSD/Ao $\leq 0.3)$ and Median $(0.3<$ VSD/Ao) group. Plasma MMP-2 and MMP-9 activities were assayed by gelatin zymography.

There was a significant higher MMP-2 activity in the VSD (Trivial, Small and Median) groups compared with that in Control group. The plasma MMP-9 activity showed a similar trend as the findings in MMP-2 activity. After one year follow-up, a significant difference in the MMP-9 activity was found between VSD spontaneous closure and non-closure groups. In conclusion, a positive trend between the severity of VSD and activities of MMP-2 and MMP-9 was found. Our data imply that MMP-2 and MMP-9 activities may play a role in the pathogenesis of VSD.
\end{abstract}

Key words: Matrix metalloproteinase-2, Matrix metalloproteinase-9, Tissue inhibitor of metalloproteinase-3, Ventricular septal defect

\section{Introduction}

Ventricular septal defect (VSD) is the most common form of congenital heart diseases (CHD) that accounts for $40 \%$ of the patients with CHD [1]. The defect can occur to any portion of the ventricular septum, and the physiologic consequences can range from trivial to severe VSD. Sometimes, VSD stands not only as an isolated cardiac malformation, but also as an intrinsic component of several complex malformations such as tetralogy of Fallot. However, our study has focused on the patients whose cardiac malformation was predominately caused by VSD.

Rates of spontaneous closure for membranous and muscular VSD in infants and children were $37 \%$ and $50 \%$, respectively, during a mean follow-up of 12 months [2]. Others don't have such a fortunate clinical course and their VSDs persist. The development of echocardiography had provided a useful tool to observe the natural processes of spontaneous VSD clo- 
sure [3]. Perimembranous defects often close by the development of a saccular pouch or aneurysm derived from tissue from the septal leaflet of tricuspid valve. Muscular defects appear to close by progressive growth of tissue from the right ventricular side of the circumference of the defect. Neither detailed molecular insight of spontaneous VSD closure, nor the associations among extracellular matrix (ECM), MMPs and spontaneous VSD closure has been clarified.

Matrix metalloproteinases (MMPs) is a family of extracellular zinc-dependent neutral endopeptidases [4] capable of degrading essentially all ECM components including fibrillar and non-fibrillar collagens, fibronectin, laminin and basement membrane glycoproteins $[5,6]$. MMPs usually play an important role in ECM remodeling in physiologic situations, such as embryonal development, tissue regeneration, and wound repair. In addition, because of their potent degradative capacities, increasingly more researchers have shifted their focus to the relationship between MMPs function and different pathological conditions.

The tissue inhibitors of metalloproteinases (TIMPs) regulate and maintain matrix homeostasis when present in the dynamic interstitial compartment. TIMPs directly inhibit the disruptive MMPs and have been implicated in the regulation of cell profile, function, and survival independent of their MMP-inhibitory effects. In light of these different biological roles, the TIMPs may play a central role in cardiac remodeling. TIMP-3 may contribute to the regulation of matrix remodeling and its reduction may promote a transition from compensated to end-stage congestive heart failure [7].

Serum MMPs activities are altered in various diseases and have been considered as potential clinical biomarkers of disease, especially for the diagnosis and/or prognosis of cardiovascular diseases. Circulating MMP-9 could be a repair indicator of VSD [8] and a biomarker to identify the patients with preserved left ventricular systolic function after AMI [9, 10]. Circulating MMP-2 is elevated in the patients with congestive heart failure (CHF) and could be used to predict the outcome in CHF patients [11, 12]. Early measurements of plasma MMP-2 could predict the infarct size and ventricular dysfunction in ST-elevation myocardial infarction [13].

MMPs are involved in regulating epithelial-to-mesenchymal transitions (EMTs) that are responsible for forming both populations of epithelial and mesenchymal cells. MMP-2 is expressed by endocardial cells prior to and during the EMT of endocardium in both the atrioventricular and outflow tract regions of developing heart $[14,15]$. MMPs may play a pivotal role and involve in the endocardial and car- diac septation, and cardiac developing [16]. Therefore, it is worth investigating the changes of circulating MMP-2 and MMP-9 on the mechanism of VSD formation or closure.

\section{Materials and Methods}

\section{Patients and plasma collection}

A total of 96 children with perimembranous VSD were recruited from the outpatient base of the Taipei and Hsinchu Maykay Memorial Hospitals. The study was performed with the approval of the Institutional Review Board of Mackay Memorial Hospital. Informed consent was obtained from each patient. All the patients with defects enrolled in this study were documented by 2-D echocardiography. The plasma of 12 healthy children donated blood samples as a control group in the MMPs activity assays.

Blood samples were collected by needle aspiration from the antecubital vein. Plasma was isolated by centrifuge $3,000 \times g$ for $10 \mathrm{~min}$, and stored at $-80^{\circ} \mathrm{C}$ for further zymography assay.

\section{Patient grouping by VSD diameter/diameter of aortic root (Ao) ratio}

Clinically, VSD can be classified into three defect sizes: large, median and small sizes. The large defect is defined when the size is larger than two-thirds of the Ao; the median size is between two-thirds and one-third of the Ao; and small if the size is less than one-third of the Ao $[17,18]$.

In clinical settings, large VSD patients with poor medical control mandatorily undergo surgery. Therefore, patients in our study were exclusively those without extremely large defects. According to the definition of VSD/Ao ratio, the patients were classified into three groups: VSD/Ao ratio $\leq 0.2$ as Trivial group; $0.2<$ VSD/Ao $\leq 0.3$ as Small group; and $0.3<$ VSD/Ao is considered as Median group.

\section{Gelatin zymography assay}

The activities of MMP-2 and MMP-9 in the plasma were measured by gelatin gel zymography as previously described $[19,20]$. The plasma was mixed with 2 x zymography sample buffer $(0.125 \mathrm{M}$ Tris- $\mathrm{HCl}$, $\mathrm{pH} 6.8,20 \%$ [v/v] glycerol, $4 \%$ [w/v] SDS, and $0.005 \%$ bromophenol blue), incubated for $10 \mathrm{~min}$, and then loaded into SDS-PAGE that was performed in 7\% acrylamide gels containing $0.1 \%(\mathrm{w} / \mathrm{v})$ gelatin (Sigma-Aldrich, St. Louis, MO, USA). After electrophoresis under power supply of $100 \mathrm{~V}$, the gel was washed twice for $30 \mathrm{~min}$ in zymography renaturing buffer (2.5\% Triton X-100) with gentle shake to remove SDS, then incubated for $18 \mathrm{~h}$ at $37^{\circ} \mathrm{C}$ in reaction buffer (50 $\mathrm{mM}$ Tris- $\mathrm{HCl}, \mathrm{pH} 7.4,200 \mathrm{mM} \mathrm{NaCl}$, and $5 \mathrm{mM}$ 
$\mathrm{CaCl}_{2}$ ). The gels were then stained with Coomossie blue for $30 \mathrm{~min}$ prior to destain with destain buffer ( $50 \%$ methanol, $10 \%$ acetic acid, and $40 \% \mathrm{dd}_{2} \mathrm{O}$ ). The presence of enzyme activity was evident by clear or unstained zones, indicating the action of the enzyme on the gelatin substrate [21]. Gelatinase activity in the gel slab was quantified by Scion Image software (NIH, Bethesda, MD, USA) that quantifies the area of bands hydrolyzed by gelatinase. An MMP-2 or MMP-9 positive control (Chemicon, Temecula, CA, USA) was separately contained in each gel as a standard intensity value to normalize the intensity of samples.

\section{Enzyme-linked immunosorbent assay}

The concentrations of TIMP-3 in the plasma were measured by ELISA. Secretion of TIMP-3 in the plasma was quantified using ELISA assays according to the manufacturer's recommendations (Abcam Inc., Cambridge, MA, USA). The samples were diluted appropriately to fall within the standard range of the assay.

\section{Statistical analysis}

The levels of various markers in the VSD samples are expressed as the mean \pm standard deviation (SD) or shown as the median (interquartile range; IQR). Data was analyzed by using SPSS for Windows (SPSS statistics package, Chicago, IL, USA). To compare the quantitative MMP activities between two groups, Student's t-test was used for presumably normally distributed variables. One-way analysis of variance (ANOVA) method was used to test the differences in the MMPs enzyme activities among groups when the group numbers were more than two. A $p$ value less than 0.05 was considered significant.

\section{Results}

\section{The information of patients}

Age and gender distribution of the 96 patients and 12 control children in this study were presented in Table 1. The mean age of all patients is $4.25 \pm 2.54$ (mean $\pm \mathrm{SD}$ ) years old, with no significant difference from the control group.

All the patients enrolled in this study were documented by 2-D echocardiography by cardiologists. The diameter of VSD was defined as VSD size, and ratio of VSD diameter and diameter of aortic root (Ao) was measured. According to the VSD/Ao ratio, patients were classified into three groups, Trivial $(\mathrm{VSD} /$ Ao ratio $=0.14 \pm 0.04)$, Small $(0.25 \pm 0.04)$ and Median $(0.43 \pm 0.01)$. Control group and each subgroup (Trivial, Small and Median) is comprised of 12, 47, 30 and 19 patients, respectively (Table 1).
Table I. VSD patients' age, gender, VSD size and VSD/Ao ratio

\begin{tabular}{lllll}
\hline & $\begin{array}{l}\text { Control } \\
\text { group } \\
(\mathrm{n}=12)\end{array}$ & $\begin{array}{l}\text { Trivial } \\
\text { group } \\
(\mathrm{n}=47)\end{array}$ & $\begin{array}{l}\text { Small } \\
\text { group } \\
(\mathrm{n}=30)\end{array}$ & $\begin{array}{l}\text { Median } \\
\text { group } \\
(\mathrm{n}=19)\end{array}$ \\
\hline Male:Female & $6: 6$ & $21: 26$ & $15: 15$ & $7: 12$ \\
Age (years) & $4.76 \pm 2.74$ & $4.06 \pm 2.26$ & $4.16 \pm 2.66$ & $4.84 \pm 2.96$ \\
VSD size $(\mathrm{mm})$ & - & $3.06 \pm 0.88$ & $4.30 \pm 0.85$ & $5.75 \pm 1.21$ \\
Ao $(\mathrm{mm})$ & & $18.70 \pm$ & $17.81 \pm$ & $14.01 \pm 3.28$ \\
& & 4.92 & 4.17 & \\
VSD/Ao ratio & - & $0.14 \pm 0.03$ & $0.25 \pm 0.04$ & $0.43 \pm 0.10$
\end{tabular}

Data were expressed as mean \pm SD. VSD, ventricular septal defect; Ao, aortic root diameter.

\section{MMPs and TIMP-3 activities in different VSD groups}

The MMP-2 activities in children with normal and different VSD groups were determined by gelatin zymography. The activities of MMP-2 enzyme in the VSD groups were compared with the Control group in Figure 1A (Median, Small, Trivial groups vs. Control group: 845 (710-945) [median (IQR)], 840 (618-1,050), 787 (604-935) ng/mL vs. 554 (407-689) $\mathrm{ng} / \mathrm{mL})$. The results revealed that the circulating MMP-2 activity was significantly increased in the VSD patients. Compared with the Control group, the MMP-2 activity of Trivial, Small and Median groups were enhanced by $1.4(p<0.01), 1.5(p<0.01)$ and 1.5 $(p<0.01)$ folds, respectively (Figure 1A). However, differences in the plasma MMP-2 activity among the VSD groups were insignificantly.

The activities of plasma MMP-9 was also determined by gelatin zymography and shown in Figure 1B (Median, Small, Trivial groups vs. Control group: 38 (32-43), 35 (26-43), 28 (19-35) ng/mL vs. 29 (25-32) $\mathrm{ng} / \mathrm{mL}$ ). A trend of increased MMP-9 activity in the VSD groups was found. However, only the MMP-9 activity in Median group was significantly higher than that in Control group $(p<0.01)$.

The concentrations of plasma TIMP-3 was also determined by ELISA (Median, Small, Trivial groups vs. Control group: 1853 (1222-2019), 1748 (1092-1827), $1800 \quad(1135-1859) \quad \mathrm{pg} / \mathrm{mL}$ vs. 1230 (1045-1462) $\mathrm{pg} / \mathrm{mL}$ ). The TIMP-3 concentrations were no significant difference among these groups.

\section{Relationships between MMPs activities and VSD defects}

Circulating MMP-2 and MMP-9 activities of all VSD patients were used to determine the relationship between MMPs activities and VSD defects defined by VSD size and VSD/Ao ratio. For the plasma MMP-2 activity, it did not show significant correlations with VSD size and VSD/Ao ratio (Figure 2A and 2B). 
Whereas, positive correlations between MMP-9 activity and VSD size $\left(\mathrm{r}^{2}=0.073, p<0.05\right)$ as well as VSD/Ao ratio $\left(\mathrm{r}^{2}=0.170, p<0.05\right)$ were evaluated (Figure 2C and 2D).

\section{MMP-2 and MMP-9 activities in patients with spontaneous closure VSD}

Patient groups were followed one year later in outpatient department and received an echocardiographic exam and general physical examination performed by a pediatric cardiologist. Totally 82 patients returned to the outpatient department according to

\section{(A)}
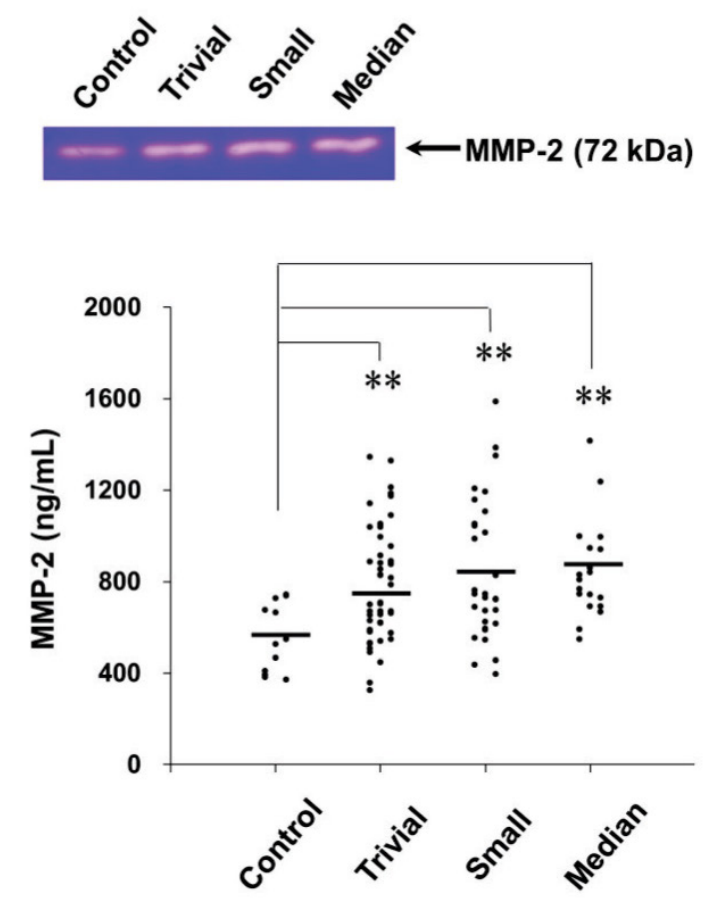

the programmed schedule. After one year follow-up, a total of 14 spontaneous closures occurred $(17 \%)$ and 68 patients remained their defects. The plasma MMP-2 activity of VSD patients in the spontaneous closure group was compared with non-closure group, and no significant difference was noted (791 (617-945) $\mathrm{ng} / \mathrm{mL}$ vs. 808 (624-990) ng/mL, $p=0.825$ ) (Figure $3 \mathrm{~A})$. In contrast, the plasma MMP-9 activity of VSD patients in the spontaneous closure group was significantly higher than non-closure group (42 (32-48) $\mathrm{ng} / \mathrm{mL}$ vs. 31 (25-38) ng/mL, $p<0.01$ ) (Figure 3B).

(B)
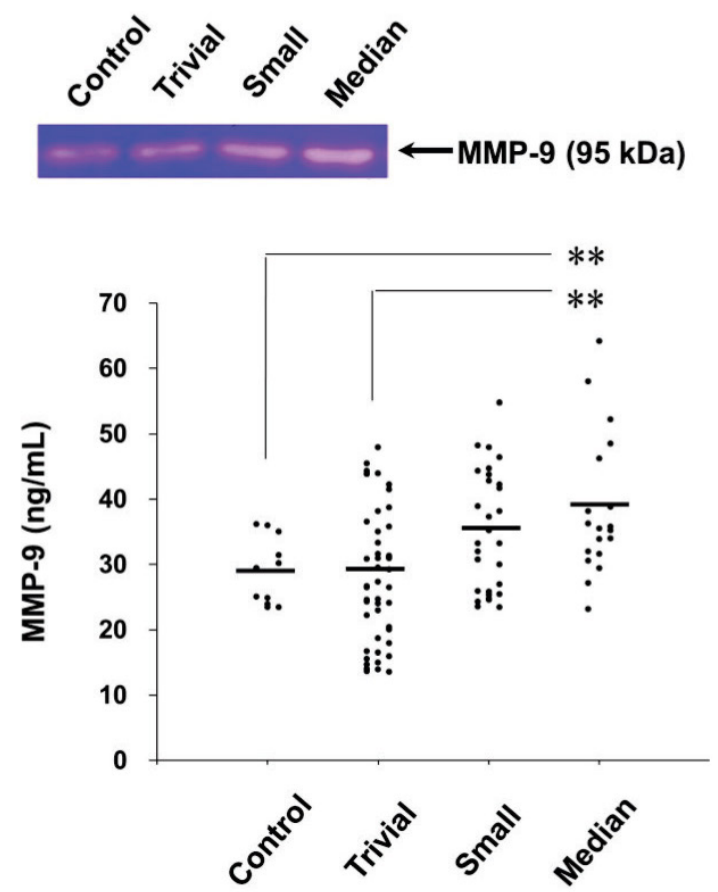

Figure I. The circulating MMP-2 and MMP-9 activities in the patients with different levels of VSD severity. The MMP-2 (A) and MMP-9 (B) activities in the patients with different VSD severity were determined by gelatin zymography. The gelatinase activities detected in this study were based on pro-MMP-2 $(72 \mathrm{kDa})$ or pro-MMP-9 $(95 \mathrm{kDa})$. Each symbol represents one individual, and horizontal bars represent mean value in each group. The compared results of the MMP-2 or MMP-9 activity in the Control $(n=12)$, Trivial $(n=47)$, Small $(n=30)$ and Median $(n=19)$ groups with one way ANOVA analysis were performed. ** indicates $p<0.01$, compared to the Control group. 
(A)

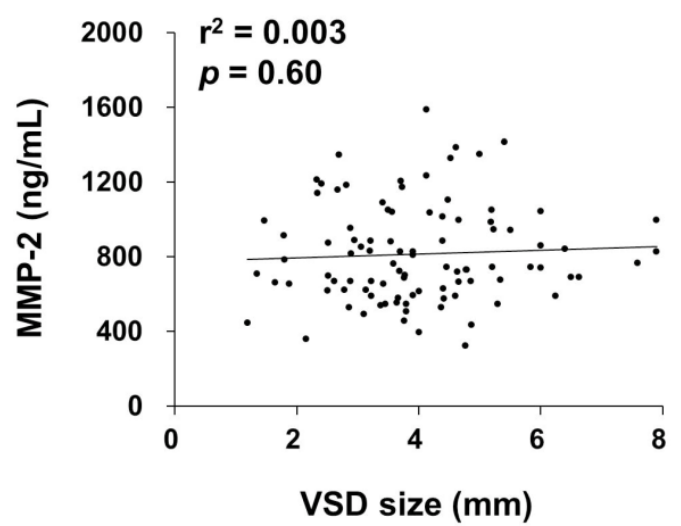

(C)

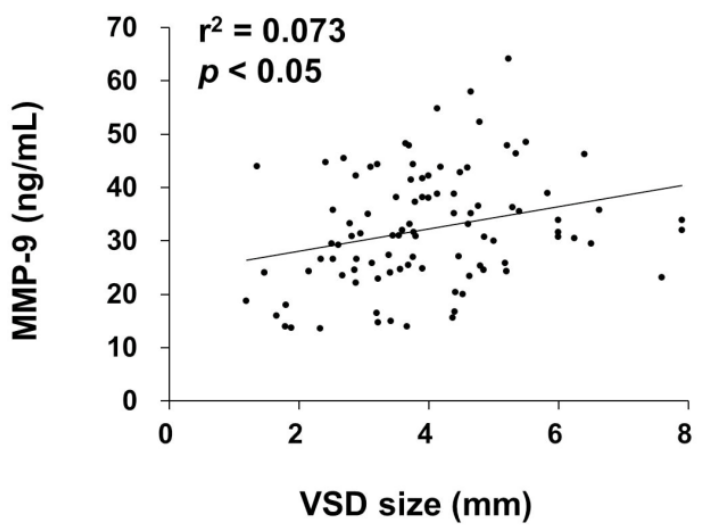

(B)

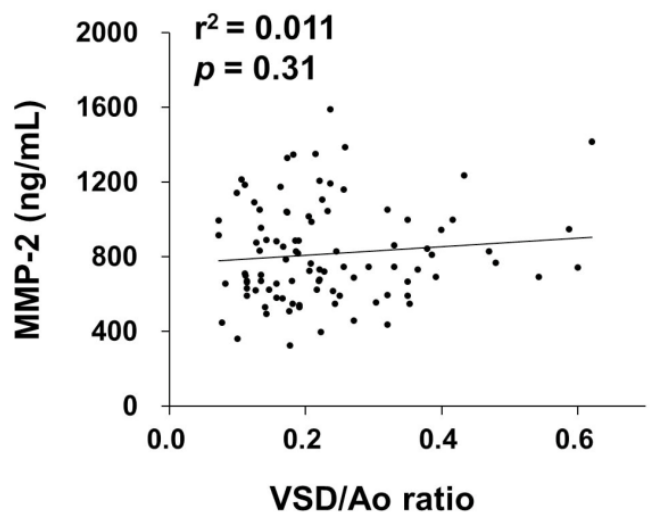

(D)

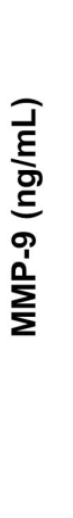

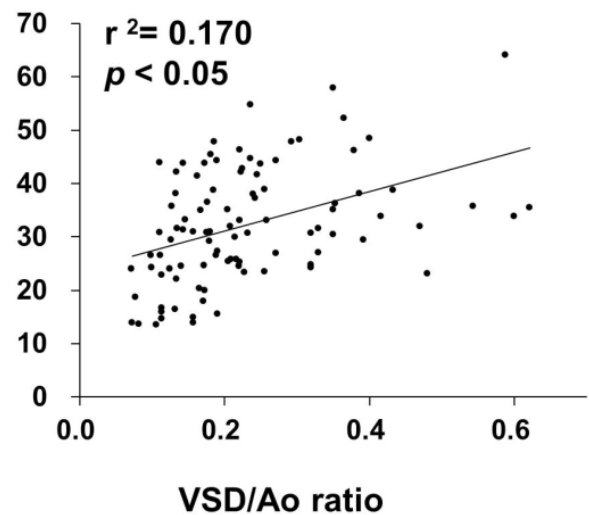

Figure 2. Correlations between circulating MMPs activities and VSD size or VSD/Ao ratio in the children patients. Pearson's correlation analysis (SPSS statistics package, Chicago, IL, USA) was applied. Plasma MMP-2 activity in the VSD patients was insignificantly correlated with the VSD size $(\mathbf{A})$ and VSD/Ao (B). Whereas, MMP-9 activity was significantly and positively correlated with the VSD defects size (C) and VSD/Ao (D) $(p<0.05)$.

\section{(A)}

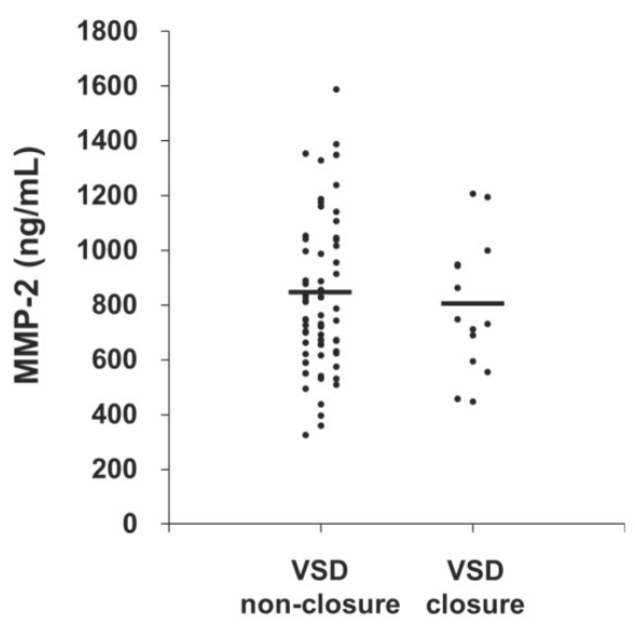

(B)

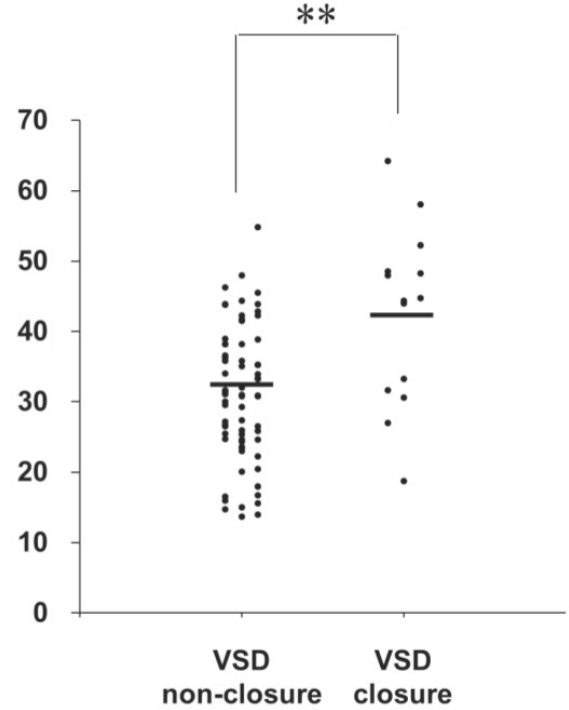

Figure 3. Circulating MMPs activities in the patients who received serial echocardiographic follow-up examinations. The MMP-2 (A) and MMP-9 (B) activities in the plasma from each VSD closure and non-closure patient were indicated, with horizontal bars representing mean values in each group. ${ }^{* *}$ indicates $p<0.01$. 


\section{Discussion}

In the present study, the VSD patients were categorized into three levels, Trivial (VSD/Ao ratio $\leq$ $0.2)$, Small $(0.2<\mathrm{VSD} / \mathrm{Ao} \leq 0.3)$ and Median (VSD/Ao > 0.3) groups, according to the examinations of 2-D echocardiography. The children patients with large VSD were excluded since surgery is mandatory for such patients with poor medical control. There is a notable finding that circulating MMP-2 and MMP-9 activities, but not circulating TIMP-3 concentrations bear a positive association with the levels of VSD severity (Figure 1 and 2). This is the first study to specifically identify the relationships between circulating MMP-2 as well as MMP-9 and severity of VSD in children patients.

The first description of MMP-like activity was related to the metamorphosis of the tadpole [22]. This and many other subsequent discoveries strongly suggest that this well-characterized family of metalloproteinases is also intimately involved in normal tissue growth and ECM remodeling [23]. Morphogenesis and tissue growth, remodeling, and repair are sentinel features of childhood and adolescence. Heart morphogenesis involves the characteristic mechanisms of cell proliferation, cell rearrangements, cell to cell and cell to matrix interactions, and tissue remodeling. Linask et al. documented that MMP activity regulates the coordination of early heart organogenesis by affecting ventral closure of the heart and ECM degradation in dorsal mesocardium [24]. Yet nowadays, only little knowledge on the production, secretion, and clearance of these important proteinases throughout normal growth and development in human heart is available. The elevated level of amniotic fluid MMP-9 has been reported in ASD cases [25]. However, no investigation addressing the changes of circulating MMPs in the children patients either with ASD or VSD has ever been documented.

In several studies, it was proposed that ventricular septal aneurysm is an important mechanism of closure and shows a more favorable prognosis in perimembranous defects $[26,27]$. In this study, the blood samples were only collected from the VSD children on the day they were diagnosed as VSD patients, and the plasma were prepared for gelatin zymography assay to test whether plasma MMP-2 and MMP-9 activity can be as prospective biomarker for VSD closure. Therefore, follow up measurements of plasma MMP-2 and MMP-9 in the VSD patients did not be investigated. The circulating MMP-2 and MMP-9 activities in the VSD patients who have spontaneous closure and non-closure have been investigated in this study. Although MMP-2 activity has no significant difference in the patients with different levels of VSD severity, MMP-9 activity showed significant difference related to the VSD closure (Figure 3 ). These data show that spontaneous closure group has a higher level of circulating MMP-9 activity. Therefore, we proposed that in vivo proteases may play a role relative to the spontaneous closure of VSD. It seemed to infringe the instincts; but with the expanding and growing data, some authors also suggest that MMPs may act as good candidates in helping physicians resolve some pathologic conditions [28, 29]. We expect great difficulties in striving to realize the interaction between MMP family and mechanism of VSD because of their intricate and complicated roles in physiology and pathogenesis. There remains an urgent need to study further on this matter.

TIMPs- 1 through -4 regulate the activity of zinc metalloproteases, TIMP-3 can form a tight complex with proMMP-2 and proMMP-9 to regulate gelatinase activities in vivo [30]. In heart diseases, TIMP-3 may contribute to the regulation of myocardial remodeling, it deficiency disrupts matrix homeostasis and causes spontaneous left ventricular dilation, cardiomyocyte hypertrophy and contractile dysfunction [7]. Our published paper proposed that the interaction between MMP-2, MMP-9 and TIMP-3 may contribute to atrial ECM remodeling of atrial fibrillation [19]. In addition, many studies also appear that the association of MMP-2, MMP-9 and TIMP-3 in heart diseases [31], heart remodeling [32, 33] and cardiocytes function properly [34], and supported that MMP-2, MMP-9 and TIMP-3 may play important role in VSD.

Some limitations of our study merit considerations. First, our sample size is fairly modest, which would primarily affect the power of our analysis to detect significant associations. This is attributed to the difficulty in collecting children's blood samples. Second, patients were recruited from only one hospital system and may not be representative of the general population. However, we used incident cases to lessen the selection bias. Nevertheless, it would be important to confirm these findings in multicenter studies. Third, it is really a difficult task to disentangle the role of each gelatinase within a single study because of its complexity of regulation in biological network. The published paper demonstrated that arterial wall tension stimulates growth and remodeling which involved in MMP-2 and MMP-9 [35]. The low shear stress circumference and reduced vessel collagen caused of postsurgical upregulated MMP-2 and MMP-9 expression in the rabbit model of artery interposed to a vein [36]. These results appear that the relationships between hemodynamic, gelatinase and remodeling, but the mechanism is still unclear. Therefore, our study revealed that MMP-2 and MMP-9 activities may play a role in the pathogenesis 
of VSD and the mechanism remains to be explored.

\section{Conclusions}

Our study results demonstrate a trend between circulating MMP-2 and MMP-9 levels and different VSD defects. We also observed an interesting association that VSD patients with spontaneous closure had higher MMP-9 level than non-closure group. It is difficult to disentangle the role of each gelatinase within a single study because of its complexity of regulation in biological network. However, this does not preclude the possibility that MMP family may have its own pathophysiological significance in VSD patients. These findings support the notion that connective tissue remodeling mediated by MMP-9 may play an important role in the mechanism of VSD formation, however, more functional investigations to determinate the influences of MMP-2 and MMP-9 activities are in need. Whether MMP-9 level provides a prediction in the ability of spontaneous closure of VSD will be assessed in additional studies.

\section{Acknowledgements}

This work was sponsored by the grants of NSC 98-2313-B-009-002-MY3 and NSC 101-2313-B-009-001MY3 from the National Science Council, Taiwan. It was also partly supported by the "Aiming for the Top University Program" of National Chiao Tung University and Ministry of Education, Taiwan (MMH-CT10201).

\section{Competing Interests}

We confirm and declare: All authors fulfilled the condition for authorship. There was no commercial support in the process of performing this study and submitting this manuscript.

\section{References}

1. Hoffman JI. Incidence of congenital heart disease: I. Postnatal incidence. Pediatr Cardiol 1995; 16:103-13.

2. Moe DG, Guntheroth WG. Spontaneous closure of uncomplicated ventricular septal defect. Am J Cardiol 1987; 60:674-8.

3. Murphy DJ Jr, Ludomirsky A, Huhta JC. Continuous-wave Doppler in children with ventricular septal defect: noninvasive estimation of interventricular pressure gradient. Am J Cardiol 1986; 57:428-32.

4. Lombard C, Saulnier J, Wallach J. Assays of matrix metalloproteinases (MMPs) activities: a review. Biochimie 2005; 87:265-72.

5. Fedarko NS, Jain A, Karadag A, Fisher LW. Three small integrin binding ligand N-linked glycoproteins (SIBLINGs) bind and activate specific matrix metalloproteinases. FASEB J 2004; 18:734-6.

6. Lin CS, Lai LP, Lin JL, et al. Increased expression of extracellular matrix proteins in rapid atrial pacing-induced atrial fibrillation. Heart Rhythm 2007; 4:938-49.

7. Fedak PW, Altamentova SM, Weisel RD, et al. Matrix remodeling in experimental and human heart failure: a possible regulatory role for TIMP-3. Am J Physiol Heart Circ Physiol 2003; 284:H626-34.

8. Tsai $\mathrm{CY}$, Lai CH, Chang MH, et al. IGF-II and MMP9 as surgical repair indicators of ventricular septal defects. Clin Chim Acta 2011; 412:761-5.

9. Miyazaki S, Kasai T, Miyauchi K, et al. Changes of matrix metalloproteinase-9 level is associated with left ventricular remodeling following acute myocardial infarction among patients treated with trandolapril, valsartan or both. Circ J 2010; 74:1158-64.

10. Kampourides N, Tziakas D, Chalikias G, et al. Usefulness of matrix metalloproteinase-9 plasma levels to identify patients with preserved left ventricular systolic function after acute myocardial infarction who could benefit from eplerenone. Am J Cardiol 2012; 110:1085-91.

11. Yamazaki T, Lee JD, Shimizu H, et al. Circulating matrix metalloproteinase-2 is elevated in patients with congestive heart failure. Eur J Heart Fail 2004; 6:41-5.

12. George J, Patal S, Wexler D, et al. Circulating matrix metalloproteinase-2 but not matrix metalloproteinase-3, matrix metalloproteinase-9, or tissue inhibitor of metalloproteinase- 1 predicts outcome in patients with congestive heart failure. Am Heart J 2005; 150:484-7.

13. Nilsson L, Hallén J, Atar D, et al. Early measurements of plasma matrix metalloproteinase-2 predict infarct size and ventricular dysfunction in ST-elevation myocardial infarction. Heart 2012; 98:31-6.

14. Alexander SM, Jackson KJ, Bushnell KM, McGuire PG. Spatial and temporal expression of the 72-kDa type IV collagenase (MMP-2) correlates with development and differentiation of valves in the embryonic avian heart. Dev Dyn 1997; 209:261-8.

15. Cai DH, Vollberg TM Sr, Hahn-Dantona E, et al. MMP-2 expression during early avian cardiac and neural crest morphogenesis. Anat Rec 2000; 259:168-79.

16. Camenisch TD, Schroeder JA, Bradley J, et al. Heart-valve mesenchyme formation is dependent on hyaluronan-augmented activation of ErbB2-ErbB3 receptors. Nat Med 2002; 8:850-5.

17. Eroğlu AG, Oztunç F, Saltik L, et al. Evolution of ventricular septal defect with special reference to spontaneous closure rate, subaortic ridge and aortic valve prolapse. Pediatr Cardiol 2003; 24:31-5.

18. Hornberger LK, Sahn DJ, Krabill KA, et al. Elucidation of the natural history of ventricular septal defects by serial Doppler color flow mapping studies. J Am Coll Cardiol 1989; 13:1111-8.

19. Chen CL, Huang SK, Lin JL, et al. Upregulation of matrix metalloproteinase-9 and tissue inhibitors of metalloproteinases in rapid atrial pacing-induced atrial fibrillation. J Mol Cell Cardiol 2008; 45:742-53.

20. Hsieh WY, Kuan TC, Cheng KS, et al. ACE/ACE2 Ratio and MMP-9 Activity as Potential Biomarkers in Tuberculous Pleural Effusions. Int J Biol Sci 2012; 8:1197-205.

21. Stawowy P, Margeta C, Kallisch H, et al. Regulation of matrix metalloproteinase MT1-MMP/MMP-2 in cardiac fibroblasts by TGF-beta1 involves furin-convertase. Cardiovasc Res 2004; 63:87-97.

22. Brinckerhoff CE, Matrisian LM. Matrix metalloproteinases: a tail of a frog that became a prince. Nat Rev Mol Cell Biol 2002; 3:207-14.

23. Stamenkovic I. Extracellular matrix remodelling: the role of matrix metalloproteinases. J Pathol 2003; 200:448-64.

24. Linask KK, Han M, Cai DH, et al. Cardiac morphogenesis: matrix metalloproteinase coordination of cellular mechanisms underlying heart tube formation and directionality of looping. Dev Dyn 2005; 233:739-53.

25. Abdallah MW, Pearce BD, Larsen N, et al. Amniotic fluid MMP-9 and neurotrophins in autism spectrum disorders: an exploratory study. Autism Res 2012; 5:428-33.

26. Freedom RM, White RD, Pieroni DR, et al. The natural history of the so-called aneurysm of the membranous ventricular septum in childhood. Circulation 1974; 49:375-84.

27. Ramaciotti C, Vetter JM, Bornemeier RA, Chin AJ. Prevalence, relation to spontaneous closure, and association of muscular ventricular septal defects with other cardiac defects. Am J Cardiol 1995; 75:61-5.

28. Hettiaratchi A, Hawkins NJ, McKenzie G, et al. The collagenase-1 (MMP-1) gene promoter polymorphism - $1607 / 2 \mathrm{G}$ is associated with favourable prognosis in patients with colorectal cancer. Br J Cancer 2007; 96:783-92.

29. Vincenti MP, Brinckerhoff CE. Signal transduction and cell-type specific regulation of matrix metalloproteinase gene expression: can MMPs be good for you? J Cell Physiol 2007; 213:355-64.

30. Nagase H, Visse R, Murphy G. Structure and function of matrix metalloproteinases and TIMPs. Cardiovasc Res 2006; 69:562-73.

31. Givvimani S, Tyagi N, Sen U, et al. MMP-2/TIMP-2/TIMP-4 versus MMP-9/TIMP-3 in transition from compensatory hypertrophy and angiogenesis to decompensatory heart failure. Arch Physiol Biochem 2010; 116:63-72.

32. Lin JM, Lai LP, Lin CS, et al. Left ventricular extracellular matrix remodeling in dogs with right ventricular apical pacing. J Cardiovasc Electrophysiol 2010; 21:1142-9.

33. Yeh CC, Malhotra D, Yang YL, et al. MEK1-induced physiological hypertrophy inhibits chronic post-myocardial infarction remodeling in mice. J Cell Biochem 2013; 114:47-55.

34. Pan $\mathrm{CH}$, Wen $\mathrm{CH}$, Lin CS. Interplay of angiotensin II and angiotensin 1-7 in the regulations of matrix metalloproteinases of human cardiocytes. Exp Physiol 2008; 93: 599-612.

35. Bayer IM, Adamson SL, Langille BL. Atrophic remodeling of the artery-cuffed artery. Arterioscler Thromb Vasc Biol 1999; 19:1499-505.

36. Feng $Y$, Shen $Y$, Zhang H. Molecular mechanism of remodeling of autologous artery graft interposed to vein in rabbit. Anat Rec (Hoboken) 2012; 295:432-7. 